\title{
THE COMPLETE CLASSIFICATION OF ASYMPTOTIC BEHAVIOR FOR BOUNDED COOPERATIVE LOTKA-VOLTERRA SYSTEMS WITH THE ASSUMPTION (SM)
}

\author{
BY \\ J. F. JIANG \\ Department of Mathematics, University of Science and Technology of China, Hefei, China
}

\begin{abstract}
Assume that the $n$-dimensional Lotka-Volterra system is cooperative with the assumption $(S M): a_{i i}<0, a_{i j}>0$ for all $i \neq j$, and all solutions of the system are bounded. Then we give a complete classification of the behavior of the solutions. This classification does not require any restriction on the auto-increase coefficients.
\end{abstract}

1. Introduction. Many mathematical models in the population ecology give rise to systems of ordinary differential equations

$$
\dot{x}_{i}=x_{i} f_{i}\left(x_{1}, x_{2}, \ldots, x_{n}\right) \quad\left(1 \leq i \leq n, x_{i} \geq 0\right)
$$

in which $x_{i}$ represents the population density of the $i$ th species and $f_{i}(x)$ represents the per capita growth rate of the $i$ th species. The system (1.1) is called cooperative if $\frac{\partial f_{i}}{\partial f_{j}} \geq 0$ for all $i \neq j$ and $x \in \mathbf{R}_{+}^{n}$. In the case that each $f_{i}$ is linear, the system (1.1) takes the following form:

$$
\dot{x}_{i}=x_{i}\left(r_{i}+\sum_{j=1}^{n} a_{i j} x_{j}\right) \quad(1 \leq i \leq n),
$$

where the parameters $r_{i}$ are called auto-increase coefficients. The system (1.2) is the classical Lotka-Volterra system which is said to be cooperative if $a_{i j} \geq 0$ for $i \neq j$. Several researchers investigated the asymptotic behavior of solutions for the cooperative system (1.2). For general $n$ and without the assumption that every auto-increase coefficient $r_{i}>$ 0 , Goh [1] used a Liapunov function to show that if (1.2) possesses a positive equilibrium and if such an equilibrium is linearly asymptotically stable (that is, if the linearized equations about the equilibrium possess a stable coefficient matrix), then it is globally asymptotically stable for initial conditions in the open positive orthant. Only when $n=2$,

Received December 24, 1994.

1991 Mathematics Subject Classification. Primary 34C11, 92A15.

Key words and phrases. Lotka-Volterra systems, cooperation, order-preserving flows, global stability, Perron-Frobenius theory. 
Goh [1] gave a complete description of the asymptotic behavior of solutions for (1.2). With the assumption that every auto-increase coefficient $r_{i}>0$, Smith [2] completely characterized the asymptotic behavior of the cooperative system (1.2). He showed that (1.2) can have at most one positive equilibrium. If none exist then all solutions with positive initial conditions tend to infinity as $t$ increases. If a positive equilibrium exists then it must be globally asymptotically stable for positive initial conditions. Meanwhile, he proved that in this case the existence of a positive equilibrium and the stability of the community matrix $A=\left(a_{i j}\right)$ are equivalent. That is, a positive steady state exists if and only if the matrix $A$ has all eigenvalues in the left half-plane. If the system (1.2) is tridiagonal, Freedman and Smith [3] have proved that every bounded solution converges to an equilibrium without any restriction on the auto-increase coefficients $r_{i}$. Under the assumption that every auto-increase coefficient $r_{i}=0$, Karakostas and Györi [4] classified the asymptotic behavior of the cooperative system (1.2). They verified that every solution to such a system converges to the origin if the principal eigenvalue $s(A)$ of $A$ is negative and that every solution with positive initial condition converges to a positive equilibrium if $s(A)=0$ and that every solution with positive initial condition tends to infinity as $t$ increases if $s(A)>0$.

The main purpose of this paper is to give a fairly complete analysis of the asymptotic behavior of solutions of the cooperative Lotka-Volterra system (1.2) under the additional assumptions that all solutions of (1.2) are bounded and $(S M): a_{i i}<0, a_{i j}>0$ for all $i \neq j$. For given auto-increase coefficients $r_{i}$ and the community matrix $A=\left(a_{i j}\right)$, we provide an algorithm for determining the asymptotic behavior of (1.2).

2. Notation and preliminaries. In this section, we establish some notational conventions and some results that will be useful in subsequent sections.

Let $x, y \in \mathbf{R}^{n}$. There is a partial order on $\mathbf{R}^{n}$ given by $x \leq y(x \ll y)$ if and only if $x_{i} \leq y_{i}\left(x_{i}<y_{i}\right)$ for $i=1,2, \ldots, n$. We reserve the notation $x<y$ for the case that $x \leq y$ and $x \neq y$. If $x \leq y,[x, y]=\{z: x \leq z \leq y\}$ and $[[x, y]]=\{z: x \ll z \ll y\}$. Let $\mathbf{R}_{+}^{n}=\{x: x \geq \mathbf{0}\}$ denote the nonnegative orthant of $\mathbf{R}^{n}$ and Int $\mathbf{R}_{+}^{n}, \partial \mathbf{R}_{+}^{n}$ be the interior and the boundary of $\mathbf{R}_{+}^{n}$ respectively. If every component of a vector $x$ is positive, we call $x$ positive. Assuming that the dimension of Euclidean space $\mathbf{R}^{n}$ is fixed we let $N=\{1,2, \ldots, n\}$. If $I \subset N$, we denote by $C(I)$ the complement of $I$ in $N$, $C(I)=N-I$. If $I \subset N$ we let $H_{I}=\left\{x \in \mathbf{R}^{n}: x_{i}=0, i \in C(I)\right\}, H_{I}^{+}=H_{I} \cap \mathbf{R}_{+}^{n}$, and Int $H_{I}^{+}=\left\{x \in H_{I}: x_{i}>0\right.$ if $\left.i \in I\right\}$. Thus, the $H_{I}^{+}, I \subset N$, make up the boundary of $\mathbf{R}_{+}^{n}$ and we sometimes refer to them as "faces". Finally, if $I \subset N, I=\left\{i_{1}<i_{2}<\cdots<i_{k}\right\}$, and $x \in \mathbf{R}^{n}$ we write $x_{I}=\left(x_{i_{1}}, x_{i_{2}}, \ldots, x_{i_{k}}\right\} \in \mathbf{R}^{k}, k=\# I$.

If $I \subset N$, then the subsystem of (1.1) obtained by setting $x_{j}=0, j \in C(I)$, will be denoted by $\left((1.1)_{\mathrm{I}}\right)$ :

$$
\dot{x}_{i}=x_{i} f_{i}\left(x_{I}\right), \quad i \in I .
$$

With this notation, (1.2) I takes the form

$$
\dot{x}_{i}=x_{i}\left(r_{i}+\sum_{j \in I} a_{i j} x_{j}\right), \quad i \in I .
$$


An $n \times n$ matrix $A=\left(a_{i j}\right)$ is called cooperative if each off-diagonal term is nonnegative and is called irreducible if it does not leave invariant any subspace of $\mathbf{R}^{n}$ spanned by a nonempty proper subset of the standard basis vectors for $\mathbf{R}^{n}$. We let $s(A)=\max \operatorname{Re} \lambda$, where $\lambda$ runs through the eigenvalues of $A$.

If the rows and columns retained of $A$ are given by subscripts

$$
1 \leq i_{1}<i_{2}<\cdots<i_{k} \leq n, \quad 1 \leq i_{1}<i_{2}<\cdots<i_{k} \leq n,
$$

then the corresponding $k \times k$ submatrix is denoted by

$$
A_{I}=A\left(\begin{array}{ll}
i_{1} & i_{2} \cdots i_{k} \\
i_{1} & i_{2} \cdots i_{k}
\end{array}\right)
$$

with $I=\left\{i_{1}<i_{2}<\cdots<i_{k}\right\}$ and its determinant is called a principal minor of $A$ with order $k$. The minors with $i_{k}=k(k=1,2, \ldots, n)$ are referred to as the leading principal minors of $A$. In this paper, we shall make use of the Perron-Frobenius theory.

Theorem 2.1 (Perron-Frobenius). Assume that $A$ is cooperative. Then

(i) $s(A)$ is an eigenvalue of $A$ (called the principal eigenvalue of $A$ ) and there exists a corresponding eigenvector (called a principal eigenvector of $A$ ) that is nonnegative. In addition, if $A$ is irreducible, then $s(A)$ is simple and to $s(A)$ there corresponds a positive eigenvector;

(ii) if $A \leq B$, then $s(A) \leq s(B)$. Furthermore, if $A$ is irreducible and $A \neq B$, then $s(A)<s(B)$

(iii) $s\left(A\left(\begin{array}{ll}i_{1} & i_{2} \cdots i_{k} \\ i_{1} & i_{2} \cdots i_{k}\end{array}\right)\right) \leq s(A)$. If $A$ is irreducible, then the inequality is strict for $k<n$;

(iv) $s(A)<0$ if and only if all leading principal minors of $-A$ are positive.

These results can be found in [5].

The system (1.1) is called cooperative (respectively, irreducible) if $f$ is $C^{1}$ and $D f(x)$ is cooperative (resp. irreducible) for each $x \in \mathbf{R}_{+}^{n}$.

The form of Kamke's result [6] we shall use is

TheOREM 2.2 (Kamke). Let (1.1) be cooperative and let $x(t), y(t)$ be solutions of (1.1) defined for $a \leq t \leq b$. Then

(i) if $x(a) \leq y(a)$, then $x(b) \leq y(b)$;

(ii) if $x(a)<y(a)$ and $x_{i}(a)<y_{i}(a)$ for $i \in I$, then $x(b)<y(b)$ and $x_{i}(b)<y_{i}(b)$ for $i \in I$.

Let $\psi_{t}(x)$ denote the solution of (1.1) passing through the initial point $x$. Sometimes, we write $\psi_{t}(x)=x(t)$; its $\omega$-limit set is denoted by $\omega(x)$. The notation $u \leq \omega(x)$ means $u \leq y$ for all $y \in \omega(x)$, and $u<\omega(x), u \ll \omega(x)$ have obvious meaning. Hirsch [7, 8] proved that if $(1.1)$ is cooperative then $\psi_{t}(x)$ is order-preserving, that is, $\psi_{t}(x) \leq \psi_{t}(y)$ whenever $x \leq y$ and $t \geq 0$ and if (1.1) is cooperative and irreducible then $\psi_{t}(x)$ is strongly order-preserving in the open positive orthant, that is, $\psi_{t}(x) \ll \psi_{t}(y)$ whenever $\mathbf{0} \ll x<y$ and $t>0$.

Theorem 2.3 (Selgrade [10]). If $(1.1)$ is cooperative and $f(p) \geq \mathbf{0}$ (resp. $\leq \mathbf{0}$ ), then every component of $\psi_{t}(p)$ is nondecreasing (resp. nonincreasing) for $t \geq 0$. In either case, if $\psi_{t}(p)$ is bounded, then its $\omega$-limit set $\omega(p)$ is an equilibrium. 
Theorem 2.4. Assume that $A$ is cooperative and irreducible and $r>\mathbf{0}$. Then the equation

$$
A x=-r
$$

has a positive solution $p \gg \mathbf{0}$ if and only if $s(A)<0$.

Proof. This result is well known. The proof given below is due to Smith and Waltman [11].

If $s(A)<0$, then $-A^{-1}=\int_{0}^{\infty} e^{A t} d t \gg \mathbf{0}$ since $e^{A t} \gg \mathbf{0}$. Therefore, Eq. (2.1) has a positive solution $p=-A^{-1} r$. Furthermore, the solution of linear ordinary differential equations

$$
\dot{x}=A x+r \quad\left(x \in \mathbf{R}_{+}^{n}\right)
$$

passing through the origin is

$$
\varphi(t)=\int_{0}^{t} e^{A(t-s)} r d s \gg \mathbf{0} \text { for } t>0 .
$$

Assume that Eq. (2.1) has a positive solution $p \gg \mathbf{0}$. Then $p$ is a steady-state solution of $(2.2)$ and, therefore, $\mathbf{0} \ll \varphi(t) \ll p$ for all $t>0$. By Theorem 2.3, (2.2) has a positive equilibrium $q \leq p$ such that $\varphi(t) \rightarrow q$ as $t \rightarrow \infty$. Let $\psi_{t}(x)$ denote the flow generated by (2.2). Then $\psi_{t}(x)$ is order-preserving, and therefore, $\psi_{t}(x) \rightarrow q$ as $t \rightarrow \infty$ for any $x \in[\mathbf{0}, q]$. Therefore, $s(A) \leq 0$. If $s(A)=0$, then the Perron-Frobenius theory implies that to $s(A)$ there corresponds a positive eigenvector $v \gg \mathbf{0}$ and $s(A)=0$ is a simple eigenvalue of $A$. Thus, the set of all solutions for $(2.1)$ is $\{q+\alpha v: \alpha \in \mathbf{R}\}$. This contradicts the fact that $[0, q]$ belongs to the basin of attraction of $q$.

REmark 1. Suppose that $r>\mathbf{0}$ and $A$ is cooperative and irreducible. Then it follows from (2.3) that there is a positive number $a>0$ such that

(i) each component of $\varphi(t)$ is strictly increasing on $[0, a]$; and

(ii) $A \varphi(t)+r>\mathbf{0}$ for any $t \in[0, a]$.

Proposition 2.5. Suppose that the community matrix $A=\left(a_{i j}\right)$ is cooperative and irreducible. If the system $(1.2)$ is bounded, then $S(A) \leq 0$.

Proof. Suppose the contrary. Then $s=s(A)>0$. From (i) of Theorem 2.1, we know that to $s$ there corresponds a positive eigenvector $v$, that is, $A v=s v$ with $v \gg \mathbf{0}$. Therefore, there exists $\eta_{0}>0$ such that $r+A(\eta v)=r+\eta s v \gg \mathbf{0}$ for all $\eta \geq \eta_{0}$. Applying Theorem 2.3, we obtain that every component of the solution $\psi_{t}(\eta v)$ of $(1.2)$ with $\eta \geq \eta_{0}$ is increasing for $t \geq 0$. We claim that such a solution $\psi_{t}(\eta v)$ is unbounded. Suppose not. Then Theorem 2.3 implies that $\psi_{t}(\eta v) \rightarrow p$ as $t \rightarrow \infty$. Since $A$ is cooperative and irreducible, the solution flow of (1.2) is strongly order-preserving in the interior of $\mathbf{R}_{+}^{n}$. Hence, $p \gg \eta v$ and $[\eta v, p]$ is contained in the basin of attraction of $p$. Let $p=\left(p_{1}, p_{2}, \ldots, p_{n}\right)$ and $\operatorname{diag}(p)$ be the diagonal matrix with $p_{i}$ 's down the diagonal. Then the linearized matrix of the right side for (1.2) at the positive equilibrium $p$ is $\operatorname{diag}(p) A$. The fact just proved above shows that $s(\operatorname{diag}(p) A) \leq 0$. For $0<\varepsilon<s$, it follows from (ii) of Theorem 2.1 that $s(\operatorname{diag}(p)(A-\varepsilon I))<s(\operatorname{diag}(p) A) \leq 0$, where $\mathbf{I}$ is the $n \times n$ identity matrix. By Theorem 2.3 of [12], we conclude that $s-\varepsilon=S(A-\varepsilon \mathbf{I})<0$, a contradiction. This proves our claim and completes the proof of this proposition. 
From Proposition 2.5 and (iii) of Theorem 2.1, we obtain that $a_{i i}<0$ for each $i$ as long as $A$ is cooperative and irreducible and all solutions of (1.2) are bounded.

In the subsequent sections, the basic assumption is that every community matrix $A_{I}$ corresponding to the subsystem $\left((1.2)_{\mathrm{I}}\right)$ is irreducible, which implies that the flow generated by $\left((1.2)_{\mathrm{I}}\right)$ is strongly order-preserving on the interior of the corresponding positive cone. Therefore, we must assume that

$$
a_{i i}<0 \quad \text { and } \quad a_{i j}>0 \text { for } i \neq j .
$$

3. The case $s(A)<0$. In this section, we shall prove the following.

Theorem 3.1. Assume that (SM) holds and that $s(A)<0$. Then $(1.2)$ has an equilibrium $p$ such that $\omega(x)=\{p\}$ for all $x \gg \mathbf{0}$. The equilibrium $p$ is either in Int $\mathbf{R}_{+}^{n}$ or in $\partial \mathbf{R}_{+}^{n}$.

Before proceeding to the proof of Theorem 3.1, we first prove two lemmas on the following auxiliary system:

$$
\dot{y}_{i}=\left(y_{i}+c_{i}\right)\left(r_{i}+\sum_{j=1}^{n} a_{i j} y_{i}\right) \quad(i=1,2, \ldots, n)
$$

where $c_{i} \geq 0$ and $y_{i} \geq 0$ for each $i$.

LEMma 3.2. Assume that (SM) holds and that the origin is an equilibrium of (3.1) (that is, $c_{i} r_{i}=0$ for each $i$ ). Then the origin of (3.1) is globally asymptotically stable in $\mathbf{R}_{+}^{n}$ if one of the following conditions holds:

(i) $r<\mathbf{0}$ and $s(A) \leq 0$;

(ii) $r=\mathbf{0}$ and $s(A)<0$.

Proof. First, we notice that the origin is an isolated equilibrium of (3.1) if either (i) or (ii) holds. Obviously, if (ii) is true, then $A$ is nonsingular and the origin is the unique equilibrium of (3.1). Suppose that $s(A) \leq 0$. Then, by (SM) and (iii) of Theorem 2.1, $s\left(A_{I}\right)<0$ for all $I \subset N=\{1,2, \ldots, n\}$ with $\# I<n$. The assumption (SM) implies that $A_{I}$ is irreducible for each $I$. Thus, $-A_{I}^{-1} \gg \mathbf{0}$ for each $I$ with $\# I<n$. If (3.1) has an equilibrium $p \in \partial \mathbf{R}_{+}^{n} \backslash\{\mathbf{0}\}$, then there is a subset $I \subset N$ such that $p_{I} \gg \mathbf{0}$ and $p_{J}=\mathbf{0}$ where $J=N-I \neq \varnothing$. It immediately follows that $r_{I}+A_{I} p_{I}=\mathbf{0}$, which implies that $p_{I}=-A_{I}^{-1} r_{I} \leq \mathbf{0}$, a contradiction to the fact that $p_{I} \gg \mathbf{0}$. This proves that (3.1) has no other equilibrium lying in $\partial \mathbf{R}_{+}^{n}$ other than the origin. Assume that (3.1) has an equilibrium sequence $\left\{p_{n}\right\}$ such that $p_{n} \gg \mathbf{0}$ and $p_{n} \rightarrow \mathbf{0}$ as $n \rightarrow \infty$. Then $A p_{n}+r=\mathbf{0}$ for each $n$. Letting $n \rightarrow \infty$, we obtain that $r=\mathbf{0}$, contradicting $r<\mathbf{0}$. This shows that the origin is an isolated equilibrium of (3.1).

Let $v \gg \mathbf{0}$ denote the principal eigenvector corresponding to $s(A)$ and let $L(v)=$ $\{\alpha v: \alpha>0\}$. For any $x=\alpha v \in L(v), r+A x=r+\alpha s(A) v<\mathbf{0}$ if either (i) or (ii) is true. Theorem 2.3 implies that $\psi_{t}(\alpha v)$ converges to an equilibrium of (3.1) as $t \rightarrow \infty$. This proves that $\omega(\alpha v)=\{\mathbf{0}\}$ for $\alpha$ sufficiently small. Set

$$
\alpha_{0}=\sup \{\alpha: \tau \in[0, \alpha], \omega(\tau v)=\{\mathbf{0}\}\} .
$$


We claim that $\alpha_{0}=\infty$. Otherwise, $\alpha_{0}<\infty$. Then $r+A\left(\alpha_{0} v\right)<\mathbf{0}$. (SM) implies that the flow $\psi_{t}(y)$ of (3.1) in the open positive orthant Int $\mathbf{R}_{+}^{n}$ is strongly order-preserving. Thus, we can easily prove that $\psi_{t}\left(\alpha_{0} v\right) \ll \alpha_{0} v$ for $t>0$ by using Theorem 2.3. Fixing $t_{0}>0$, we have $\psi_{t_{0}}\left(\alpha_{0} v\right) \in\left[\left[\mathbf{0}, \alpha_{0} v\right]\right]$. Because $\psi_{t_{0}}(y)$ is continuous with respect to $y$, there exists an open set $U$ containing $\alpha_{0} v$ such that $\psi_{t_{0}}(y) \in\left[\left[\mathbf{0}, \alpha_{0} v\right]\right]$ for any $y \in U$. By the definition of $\alpha_{0}$ and the order-preserving property of the flow $\psi_{t}(y), \omega(y)=\{\mathbf{0}\}$ for any $y \in\left[\mathbf{0}, \alpha_{0} v\right]$. Therefore, for $\alpha>\alpha_{0}$ sufficiently close to $\alpha_{0}, \omega(\alpha v)=\{\mathbf{0}\}$, contradicting the definition of $\alpha_{0}$. This contradiction shows that $\omega(\alpha v)=\{\mathbf{0}\}$ for any $\alpha>0$. For any $y>\mathbf{0}$, there exists an $\alpha>0$ such that $\mathbf{0}<y \ll \alpha v$. The order-preserving property implies that $\mathbf{0}<\psi_{t}(y) \ll \psi_{t}(\alpha v)$ for $t>0$; thus, $\omega(y)=\{\mathbf{0}\}$. This completes the proof.

LEMmA 3.3. Assume that $r>\mathbf{0}$. Then

(i) if $s(A) \geq 0$, then the solution $\psi_{t}(y)$ of (3.1) tends to infinity as $t$ increases for any $y \in \operatorname{Int} \mathbf{R}_{+}^{n}$;

(ii) if $s(A)<0$, then (3.1) has a positive equilibrium that is globally asymptotically stable for positive initial conditions.

Proof. Let $G(y)$ denote the right side of (3.1). Then (SM) implies that $A=\left(a_{i j}\right)$ is cooperative and irreducible. From Remark 1, we obtain that there is a $C^{1}$ curve $C$ defined by $y=\varphi(t)$ for $t \in[0, a]$ satisfying that $\varphi(0)=\mathbf{0}, \varphi(t) \geq \mathbf{0}$, and $A \varphi(t)+r>\mathbf{0}$ for $t \in(0, a]$. Therefore, for any $y \in C$ with $y \neq \mathbf{0}, G(y)>\mathbf{0}$. Theorem 2.3 implies that each component of $\psi_{t}(y)$ is nondecreasing for $t \geq 0$ and $y \in C$. Thus, as $t$ increases, either $\psi_{t}(y)$ tends to infinity or $\psi_{t}(y)$ converges to a positive equilibrium of (3.1).

Assume that $s(A) \geq 0$. It follows from $r>\mathbf{0}$ and Theorem 2.4 that (3.1) has no positive equilibrium. This shows that $\psi_{t}(y)$ tends to infinity as $t$ increases for any $y \in C$ with $y \neq \mathbf{0}$. For any $x \gg \mathbf{0}$, there exists a point $y \in C$ with $\mathbf{0} \ll y \ll x$. The assumption $(\mathrm{SM})$ and the Kamke theorem imply that $\psi_{t}(y) \ll \psi_{t}(x)$ for $t>0$. Hence $\psi_{t}(x)$ also tends to infinity as $t$ increases.

Assume that $s(A)<0$. By $(\mathrm{SM})$ and Theorem 2.4, (3.1) has a unique positive equilibrium $p$. Together with the fact proved in the first paragraph, we obtain that $\psi_{t}(y)$ converges to $p$ as $t \rightarrow \infty$ for any $y \in C$. Let $v$ denote the principal eigenvector of $A$ and write $L(v)=\{p+\alpha v: \alpha>0\}$. Then, for any $x=p+\alpha v \in L(v)$, $r+A x=r+A p+A(\alpha v)=\alpha s(A) v \ll \mathbf{0}$, that is, $G(x) \ll \mathbf{0}$. Applying Theorem 2.3 to such an $x$, we conclude that $\psi_{t}(x)$ converges to $p$ as $t \rightarrow \infty$. For any $z \in \operatorname{Int} \mathbf{R}_{+}^{n}$, there are two points $y \in C$ and $x \in L(v)$ such that $y \ll z \ll v$. The Kamke theorem implies that $\psi_{t}(y) \ll \psi_{t}(z) \ll \psi_{t}(x)$ for $t>0$. Since $\psi_{t}(x)$ and $\psi_{t}(y)$ are convergent to $p, \psi_{t}(z)$ is also convergent to $p$. This proves the lemma.

Proof of Theorem 3.1. Let $F(x)$ denote the right side vector field of (1.2). Since $s(A)<0, r+A(\alpha v)=r+\alpha s(A) v \ll \mathbf{0}$ for $\alpha$ sufficiently large. Theorem 2.3 implies that $\mathbf{0} \ll \psi_{t}(\alpha v) \ll \alpha v$ for $t>0$ and such an $\alpha v$. Using this fact and the order-preserving property, we can easily prove that all solutions of (1.2) are bounded if $s(A)<0$.

Suppose $r>\mathbf{0}$. Then (ii) of Lemma 3.3 with $c_{1}=c_{2}=\cdots=c_{n}=0$ implies that (1.2) has a unique equilibrium such that it attracts all positive initial conditions. Suppose $r \leq \mathbf{0}$. Then Lemma 3.2 with $c_{1}=c_{2}=\cdots=c_{n}$ implies that the origin is globally asymptotically stable in $\mathbf{R}_{+}^{n}$. 
The remaining case to consider is that there are two nonempty index subsets $I_{0}, J_{0} \subset$ $N$ such that $N=I_{0} \cup J_{0}$ with $r_{I_{0}}>\mathbf{0}$ and $r_{J_{0}} \ll \mathbf{0}$. Now, we first study the subsystem $(1.2)_{\mathrm{I}_{0}}$ of $(1.2)$ :

$$
\dot{x}_{i}=x_{i}\left(r_{i}+\sum_{j \in I_{0}} a_{i j} x_{j}\right), \quad i \in I_{0} .
$$

If we express $I_{0}=\left\{i_{1}<i_{2}<\cdots<i_{k}\right\}$, then the community matrix of $(1.2)_{\mathrm{I}_{0}}$ is

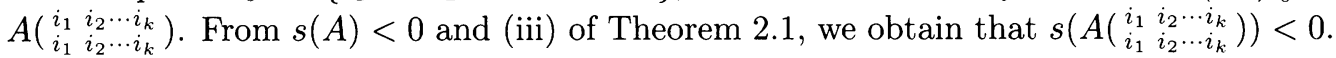
Applying (ii) of Lemma 3.3 to $(1.2)_{\mathrm{I}_{0}}$ with $c_{i}=0$ for $i \in I_{0}$ and $r_{I_{0}}>\mathbf{0}$, we can conclude that $(1.2)_{\mathrm{I}_{0}}$ has a unique positive equilibrium $u^{*} \in \operatorname{Int} \mathbf{R}_{+}^{k}$ such that it attracts all positive initial conditions in $\mathbf{R}_{+}^{k}$. We define a point $p \in \partial \mathbf{R}_{+}^{n}$ given by $p_{j}=u_{j}^{*}>0$ for $j=i_{j}$ $(1 \leq j \leq k)$ and $p_{j}=0$ for $j \in J_{0}$. Then it is easy to see that $p$ is an equilibrium of (1.2). $\partial \mathbf{R}_{+}^{n}$ contains at most $2^{n}-1$ equilibria of (1.2). Let $E$ denote the set of all equilibria for (1.2). For $p \in E$, we define $\sigma(p)=\#\left\{i: p_{i}>0\right\}$ and $m=\max \left\{\sigma(p): p \in \partial \mathbf{R}_{+}^{n} \cap E\right\}$. Then $k \leq m<n$. By the definition of $m$, there exists an index subset $I \subset N$ such that $\# I=m$ and $(1.2)_{\mathrm{I}}$ has a positive equilibrium $c^{*}$. Define the equilibrium $c$ of (1.2) by $c_{i}=c_{i}^{*}$ for $i \in I$ and $c_{i}=0$ for $i \in C(I)$. Now, we make a translation transformation

$$
y=x-c .
$$

Thus, (1.2) is transformed into the following form:

$$
\begin{aligned}
& \dot{y}_{i}=\left(y_{i}+c_{i}^{*}\right) \sum_{j=1}^{n} a_{i j} y_{j}, \quad \text { for } i \in I, \\
& \dot{y}_{k}=y_{k}\left(r_{k}^{*}+\sum_{j=1}^{n} a_{k j} y_{j}\right), \quad \text { for } k \in C(I),
\end{aligned}
$$

where $r_{k}^{*}=r_{k}+\sum_{j \in I} a_{k j} c_{j}^{*}$ for $k \in C(I)$. We claim that either $r_{k}^{*} \geq 0$ for each $k \in C(I)$ or $r_{k}^{*} \leq 0$ for each $k \in C(I)$.

Suppose the contrary. Then there exist $k_{1}, k_{2} \in C(I)$ such that $r_{k_{1}}^{*}>0$ and $r_{k_{2}}^{*}<0$. Let $\tilde{r}$ denote the vector defined by $\tilde{r}_{i}=0$ if $i \in I$ and $\tilde{r}_{j}=r_{j}^{*}$ if $j \in C(I)$. By the supposition, there is an index subset $I_{1} \supset I$ such that $\# I_{1}>\# I$ and $\tilde{r}_{I_{1}}>\mathbf{0}$. (ii) of Lemma 3.3 implies that $(3.3)_{\mathrm{I}_{1}}$, which is obtained in the same manner as $(1.2)_{\mathrm{I}}$, has a positive equilibrium $d^{*}$. From (3.2), $x=c+d$ is an equilibrium of (1.2) where $d=\left(d_{1}, d_{2}, \ldots, d_{n}\right), d_{i}=d_{i}^{*}$ if $i \in I_{1}$ and $d_{i}=0$ if $i \in C\left(I_{1}\right)$. Clearly, $d_{i}>0$ for each $i \in I_{1}$. Therefore, $\sigma(c+d) \geq \# I_{1}>\# I=m$, contradicting the definition of $m$. This proves our claim, that is, either $\tilde{r}>\mathbf{0}$ or $\tilde{r} \leq \mathbf{0}$.

If $\tilde{r}>\mathbf{0}$, then, applying (ii) of Lemma 3.3 to (3.3), we get that (3.3) has a positive equilibrium. Hence, from (3.2), (1.2) also has a positive equilibrium $p$. Using the global stability result of Goh [1] (indeed, we can give an independent proof here, for brief, we omit it), we can conclude that such a $p$ is globally asymptotically stable in Int $\mathbf{R}_{+}^{n}$.

If $\tilde{r} \leq \mathbf{0}$, then, from Lemma 3.2, we deduce that $y(t) \rightarrow \mathbf{0}$ as $t \rightarrow \infty$ where $y(t)$ is the solution of (3.3) passing through $y \geq \mathbf{0}$. Correspondingly, every solution $\psi_{t}(x)$ of $(1.2)$ tends to $c$ as $t \rightarrow \infty$ for all $x \geq c$. For any $x \gg \mathbf{0}$, there exists $x_{1} \in \operatorname{Int} H_{I}^{+}$and $x_{2} \gg c$ such that $x_{1} \ll x \ll x_{2}$. The Kamke theorem implies that $\psi_{t}\left(x_{1}\right) \ll \psi_{t}(x) \ll \psi_{t}\left(x_{2}\right)$ for 
$t>0$. From $\omega\left(x_{i}\right)=\{c\}$ for $i=1,2$ and the order-preserving property, we conclude that $\omega(x)=\{c\}$. The proof is complete.

From the proof of Theorem 3.1, we can obtain the following proposition.

Proposition 3.4. Let $s(A)<0$ and let (SM) hold. Assume (1.2) I has a positive equilibrium $c^{*}$. Then

(i) if $r_{k}^{*}=r_{k}+\sum_{j \in I} a_{k j} c_{j}^{*} \leq 0$ for each $k \in C(I)$, then the equilibrium $c$ of $(1.2)$ attracts all positive initial conditions in $\mathbf{R}_{+}^{n}$;

(ii) if $r_{k}^{*}=r_{k}+\sum_{j \in I} a_{k j} c_{j}^{*} \geq 0$ for each $k \in C(I)$, and $r_{k_{0}}^{*}>0$ for some $k_{0} \in C(I)$, then (1.2) has a positive equilibrium that attracts all positive initial conditions.

The advantage of Proposition 3.4 is that one can obtain the asymptotic behavior of higher-dimensional Lotka-Volterra equations with the help of lower-dimensional LotkaVolterra equations and the parameters $r_{k}^{*}$.

REMARK 3.1. From (ii) of Proposition 3.4 and the proof of Theorem 3.1, we know that if $s(A)<0$ and (SM) holds then the necessary condition for (1.2) to have a positive equilibrium is that (1.2) has an equilibrium lying in the interior of some $(n-1)$-dimensional face.

4. The case $s(A)=0$. In this section, we agree that $v$ and $w$ are the principal eigenvectors of $A$ and $A^{\mathrm{T}}$ respectively and that $\sum_{i=1}^{n} v_{i}=\sum_{i=1}^{n} w_{i}=1$, where $A^{\mathrm{T}}$ is the transpose of $A$. Our main result of this section is stated as follows.

TheOREM 4.1. Assume that $s(A)=0$ and that (SM) holds. Then exactly one of the following holds:

(i) the solution $\psi_{t}(x)$ tends to infinity as $t$ increases for $x \gg \mathbf{0}$;

(ii) (1.2) has an equilibrium $p \in \partial \mathbf{R}_{+}^{n}$ attracting all positive initial conditions;

(iii) there exists an equilibrium $p \in \partial \mathbf{R}_{+}^{n}$ such that the set of positive equilibria of (1.2) is $E_{+}=\{p+\alpha v: \alpha>0\}$. For a given $x \gg \mathbf{0}$, there exists a unique $\alpha>0$ such that $\omega(x)=\{p+\alpha v\}$, where $\alpha$ is the positive root of the following algebraic equation:

$$
\prod_{i=1}^{n}\left(p_{i}+\alpha v_{i}\right)^{w_{i}}=\prod_{i=1}^{n} x_{i}^{w_{i}}
$$

in which $p=\left(p_{1}, p_{2}, \ldots, p_{n}\right), x=\left(x_{1}, x_{2}, \ldots, x_{n}\right), v=\left(v_{1}, v_{2}, \ldots, v_{n}\right)$, and $w=$ $\left(w_{1}, w_{2}, \ldots, w_{n}\right)$.

Proof. If $r>\mathbf{0}$, then (i) of Lemma 3.3 implies that (i) of this theorem occurs. If $r<\mathbf{0}$, then, by (i) of Lemma 3.2, the origin is globally asymptotically stable in $\mathbf{R}_{+}^{n}$. If $r=\mathbf{0}$, it is easy to see that the set of all positive equilibria of (1.2) is $E_{+}=\{\alpha v: \alpha>0\}$. From Hirsch [9, Theorem 9.7] and its proof, we can conclude that every solution $\psi_{t}(x)$ passing through a positive initial point $x$ converges to an equilibrium in $E_{+}$. Actually, this case was considered by Karakostas and Györi [4] (see [4, Theorem 4.2]).

It remains to consider the case that auto-increase coefficients $r_{i}$ change sign, that is, there exists an index $I_{0} \subset N$ such that $r_{I_{0}}>\mathbf{0}$ and $r_{J_{0}} \ll \mathbf{0}$ where $J_{0}=C\left(I_{0}\right) \neq \varnothing$. Since $s(A)=0$ and $(\mathrm{SM})$ is satisfied, it follows from (iii) of Theorem 2.1 that $s\left(A\left(\begin{array}{l}i_{1} i_{2} \cdots i_{k} \\ i_{1} i_{2} \cdots i_{k}\end{array}\right)\right)<0$ for any $k<n$. Hence, all equilibria of (1.2) lying in $\partial \mathbf{R}_{+}^{n}$ are finite and applying (ii) of 
Lemma 3.3 to $(1.2)_{\mathrm{I}_{0}}$, we know that (1.2) has at least one equilibrium in $\partial \mathbf{R}_{+}^{n} \backslash\{\mathbf{0}\}$. Let $m=\max \left\{\sigma(p): p \in \partial \mathbf{R}_{+}^{n} \cap E\right\}$. Then $\# I_{0} \leq m<n$. By the definition of $m$, we can pick an equilibrium $c \in \partial \mathbf{R}_{+}^{n}$ such that $\sigma(c)=m$, that is, there is an index subset $I \subset N$ such that $\# I=m$ and $c_{i}>0$ for $i \in I$ and $c_{j}=0$ for $j \in C(I)$. Now, we make the translation transformation (3.2) that transforms (1.2) into (3.3). By arguing as in the proof of Theorem 3.1 we obtain that all $r_{k}^{*}=r_{k}+\sum_{j \in I} a_{k j} c_{j}^{*}(k \in C(I))$ do not change sign. Therefore, one of the following holds:

(a) each $r_{k}^{*} \geq 0$ and $r_{k_{0}}^{*}>0$ for some $k_{0} \in C(I)$;

(b) each $r_{k}^{*} \leq 0$ and $r_{k_{0}}^{*}<0$ for some $k_{0} \in C(I)$;

(c) each $r_{k}^{*}=0$.

If (a) occurs, then, by (i) of Lemma 3.3, every solution of (3.3) with positive initial point tends to infinity as $t$ increases. Correspondingly, all solutions $\psi_{t}(x)$ of $(1.2)$ with $x \gg c$ tend to infinity as $t$ increases. Applying Theorem (3.1) to (1.2) $\mathrm{I}$, we can deduce that $\psi_{t}(x) \rightarrow c$ as $t \rightarrow \infty$ for all $x \in \operatorname{Int} H_{I}^{+}$. For any $x \gg \mathbf{0}$, there is an $x_{1} \in \operatorname{Int} H_{I}^{+}$ such that $x_{1} \ll x$. The Kamke theorem implies that $\psi_{t}\left(x_{1}\right) \ll \psi_{t}(x)$ for $t>0$. Since the stable manifold of $c$ is $(n-1)$-dimensional, which lies in Int $H_{I}^{+}$and the unstable manifold is one-dimensional, which is tangent to $v$ at $c, c$ is a saddle and therefore, all solutions $\psi_{t}(x)$ with $x \gg \mathbf{0}$ will enter $c+\operatorname{Int} \mathbf{R}_{+}^{n}$. This proves that all solutions with positive initial conditions tend to infinity as $t$ increases.

If (b) occurs, then (i) of Lemma 3.2 implies that the origin of (3.3) is globally asymptotically stable in $\mathbf{R}_{+}^{n}$. Correspondingly, all solutions $\psi_{t}(x)$ of $(1.2)$ with $x \geq c$ converge to $c$. For any $x \gg \mathbf{0}$, there are $x_{1} \in \operatorname{Int} H_{I}^{+}$and $x_{2} \geq c$ such that $x_{1} \ll x \ll x_{2}$. The Kamke theorem implies that $\psi_{t}\left(x_{1}\right) \ll \psi_{t}(x) \ll \psi_{t}\left(x_{2}\right)$ for $t>0$. Applying Theorem 3.1 to $(1.2)_{\mathrm{I}}$, we conclude that $\psi_{t}\left(x_{1}\right) \rightarrow c$ as $t \rightarrow \infty$. Thus, together with the fact that $\psi_{t}\left(x_{2}\right) \rightarrow c$ as $t \rightarrow \infty$, we have $\psi_{t}(x) \rightarrow c$ as $t \rightarrow \infty$.

Assume that case (c) occurs. Then it is easy to see that all positive equilibria of (1.2) are in $E_{+}=\{c+\alpha v: \alpha>0\}$. In order to determine which equilibrium the solution $\psi_{t}(x)$ converges to for a given $x \gg \mathbf{0}$, we shall study the invariant function of (1.2) in this case.

Since $w$ is the principal eigenvector of $A^{\mathrm{T}}$, we have $A^{\mathrm{T}} w=\mathbf{0}$, that is,

$$
\sum_{i=1}^{n} a_{i j} w_{i}=0 \quad \text { for } j \in N
$$

In the following, we shall prove that $H: \operatorname{Int} \mathbf{R}_{+}^{n} \rightarrow(0, \infty)$ defined by

$$
H(x)=\prod_{i=1}^{n} x_{i}^{w_{i}}
$$

is an invariant function of (1.2). From $r+A(c+\alpha v)=r+A c=\mathbf{0}$ for each $\alpha>0$, we obtain that

$$
r_{i}=-\sum_{j=1}^{n} a_{i j} c_{j} \quad \text { for } i \in N
$$


Differentiating $\ln H(x)$ along a solution of (1.2) we find that

$$
\begin{aligned}
\frac{d \ln H(x)}{d t} & =\sum_{i=1}^{n} w_{i}\left(r_{i}+\sum_{j=1}^{n} a_{i j} x_{j}\right) \\
& =\sum_{i=1}^{n} w_{i} r_{i}+\sum_{j=1}^{n} x_{j} \sum_{i=1}^{n} a_{i j} w_{i} \\
& =\sum_{i=1}^{n} w_{i} r_{i} \\
& =-\sum_{i=1}^{n} w_{i} \sum_{j=1}^{n} a_{i j} c_{j} \\
& =-\sum_{j=1}^{n} c_{j} \sum_{i=1}^{n} a_{i j} w_{i}=0,
\end{aligned}
$$

where the third and the sixth equalities follow from (4.2) and the fourth equality follows from (4.3). Therefore, $\ln H(x)$, and whence $H(x)$, is an invariant function of (1.2). Now, we define a function $G:[0, \infty) \rightarrow[0, \infty)$ given by

$$
G(\alpha)=\prod_{i=1}^{n}\left(c_{i}+\alpha v_{i}\right)^{w_{i}}
$$

which is the restriction of $H$ on $E_{+}$. Since $c \in \partial \mathbf{R}_{+}^{n}, G(0)=0$. Obviously, $G^{\prime}(\alpha)>0$ for all $\alpha>0$. Hence, $G$ is strictly increasing on $[0, \infty)$ and $G(\infty)=\lim _{\alpha \rightarrow \infty} G(\alpha)=\infty$. This shows that for any constant $\tau>0$ there exists a unique $\alpha>0$ such that $\tau=G(\alpha)$, which implies that $\alpha=G^{-1}(\tau)$ where $G^{-1}$ denotes the inverse function of $G$.

For any $x^{0} \gg \mathbf{0}, H\left(\psi_{t}\left(x^{0}\right)\right) \equiv H\left(x^{0}\right)$ for $t \geq 0$. Therefore, for any $y \in \omega\left(x^{0}\right)$, $H(y)=H\left(x^{0}\right)>0$. This shows that $y \gg \mathbf{0}$, that is, $\omega\left(x^{0}\right) \gg \mathbf{0}$. Since $c$ attracts all initial conditions in Int $H_{I}^{+}$and for any $x^{0} \gg \mathbf{0}$, there exists an $x_{1} \in \operatorname{Int} H_{I}^{+}$with $x_{1} \ll x^{0}, \omega\left(x^{0}\right) \geq \omega\left(x_{1}\right)=\{c\}$. Thus, $y>c$ for any $y \in \omega\left(x^{0}\right)$. Let $u=\inf \left\{z \in E_{+}\right.$: $\left.z \geq \omega\left(x^{0}\right)\right\}$. If $u \in \omega\left(x^{0}\right)$, then $\psi_{t}\left(x^{0}\right)$ will enter $c+\operatorname{Int} \mathbf{R}_{+}^{n}$ for $t$ sufficiently large. Thus, Theorem 9.7 of [9] implies that $\omega\left(x^{0}\right)=\{u\}$. If $u \notin \omega\left(x^{0}\right)$, then $\omega\left(x^{0}\right)<u$. Since the $\psi_{t}(x)$ is strongly order-preserving in Int $\mathbf{R}_{+}^{n}, \omega\left(x^{0}\right) \ll u$; but then $u$ is not the least upper bound in $E_{+}$, a contradiction. This proves $\omega\left(x^{0}\right)$ is a singleton and $\omega\left(x^{0}\right) \in E_{+}$ for any $x^{0} \gg \mathbf{0}$. Therefore, $\psi_{t}\left(x^{0}\right)$ converges to the equilibrium $c+\alpha v$ that belongs to the hypersurface $H(x)=H\left(x^{0}\right)$, that is, $\alpha$ is the positive root of the equation

$$
\prod_{i=1}^{n}\left(c_{i}+\alpha v_{i}\right)^{w_{i}}=\prod_{i=1}^{n}\left(x_{i}^{0}\right)^{w_{i}} \text {. }
$$

REMARK 4. If $r=\mathbf{0}$, then $c=\mathbf{0}, G(\alpha)=\alpha \prod_{i=1}^{n} v_{i}^{w_{i}}$, and $G^{-1}(\tau)=\tau \prod_{i=1}^{n} v_{i}^{-w_{i}}$. Therefore, $\psi_{t}\left(x^{0}\right) \rightarrow \prod_{i=1}^{n}\left(x_{i}^{0} / v_{i}\right)^{w_{i}} v$ as $t \rightarrow \infty$. This is Theorem 4.2 of Karakostas and Györi [4].

From the proof of Theorem 4.1, the following proposition can be obtained.

Proposition 4.2. Let (SM) hold and $s(A)=0$. Assume that (1.2) I has a positive equilibrium $c^{*}$. Then exactly one of the following holds: 
(i) if $r_{k}^{*}=r_{k}+\sum_{j \in I} a_{k j} c_{j}^{*} \geq 0$ for each $k \in C(I)$ and $r_{k_{0}}^{*}>0$ for some $k_{0} \in C(I)$, then every solution of (1.2) with a positive initial condition tends to infinity as $t$ increases;

(ii) if $r_{k}^{*} \leq 0$ for each $k \in C(I)$ and $r_{k_{0}}^{*}<0$ for some $k_{0} \in C(I)$, then $c$ attracts all positive initial conditions, where $c=\left(c_{1}, c_{2}, \ldots, c_{n}\right)$ with $c_{i}=c_{i}^{*}$ for $i \in I$ and $c_{j}=0$ for $j \in C(I)$;

(iii) if $r_{k}^{*}=0$ for each $k \in I$, then the solution $\psi_{t}(x)$ with $x \gg \mathbf{0}$ converges to $c+\alpha v$ where $c$ is given in (ii) and $\alpha$ is the positive root of the algebraic equation

$$
\prod_{i=1}^{n}\left(c_{i}+\alpha v_{i}\right)^{w_{i}}=\prod_{i=1}^{n} x_{i}^{w_{i}}
$$

5. The lower-dimensional Lotka-Volterra systems. In this section, we shall give a complete description of the asymptotic behavior of the lower-dimensional system (1.2) in the parameters $a_{i j}$ and $r_{i}$. Let $X_{i}^{+}$denote the nonnegative $x_{i}$-axis. First, we consider the two-dimensional system:

$$
\begin{aligned}
& \dot{x}_{1}=x_{1}\left(r_{1}+a_{11} x_{1}+a_{12} x_{2}\right), \\
& \dot{x}_{2}=x_{2}\left(r_{2}+a_{11} x_{1}+a_{12} x_{2}\right),
\end{aligned}
$$

where $a_{12}, a_{21}>0$. Apparently, $s(A)<0$ if and only if

$$
a_{11}<0, \quad a_{22}<0, \quad \text { and } \quad a_{11} a_{22}-a_{12} a_{21}>0 .
$$

The possible equilibria of (5.1) in $\mathbf{R}_{+}^{n}$ are $E_{0}, E_{1}, E_{2}$, and $E_{*}$ given by $E_{0}=(0,0), E_{1}=$ $\left(-\frac{r_{1}}{a_{11}}, 0\right), E_{2}=\left(0,-\frac{r_{2}}{a_{22}}\right)$, and $E_{*}=\left(x_{1}^{*}, x_{2}^{*}\right)$ where

$$
x_{1}^{*}=\frac{r_{2} a_{12}-r_{1} a_{22}}{a_{11} a_{22}-a_{12} a_{21}} \quad \text { and } \quad x_{2}^{*}=\frac{r_{1} a_{21}-r_{2} a_{11}}{a_{11} a_{22}-a_{12} a_{21}} .
$$

TheOREM 5.1. Suppose that the system (5.1) satisfies (5.2). Then exactly one of the following holds.

(i) If $r>\mathbf{0}$, then $\omega(x)=\left\{E_{*}\right\}$ for $x \gg \mathbf{0}, \omega(x)=\left\{E_{i}\right\}$ for $x \in X_{i}^{+} \backslash\{\mathbf{0}\}$ in the case $r_{i}>0$, and $\omega(x)=\{\mathbf{0}\}$ for $x \in X_{i}^{+}$in the case $r_{i}=0$.

(ii) If $r \leq \mathbf{0}$, then $\omega(x)=\{\mathbf{0}\}$ for $x \geq \mathbf{0}$.

(iii) Assume $r_{1}>0$ and $r_{2}<0$. If $r_{1} a_{21}>r_{2} a_{11}$, then $\omega(x)=\left\{E_{*}\right\}$ for $x \gg \mathbf{0}$; $\omega(x)=\left\{E_{1}\right\}$ for $x \in X_{1}^{+} \backslash\{\mathbf{0}\}$ and $\omega(x)=\{\mathbf{0}\}$ for $x \in X_{2}^{+}$. If $r_{1} a_{21} \leq r_{2} a_{11}$, then $\omega(x)=\left\{E_{1}\right\}$ for $x=\left(x_{1}, x_{2}\right)$ with $x_{1}>0$ and $\omega(x)=\{\mathbf{0}\}$ for $x \in X_{2}^{+}$.

(iv) Assume that $r_{1}<0$ and $r_{2}>0$. If $r_{2} a_{12}>r_{1} a_{22}$, then $\omega(x)=\left\{E_{*}\right\}$ for $x \gg \mathbf{0}$; $\omega(x)=\left\{E_{2}\right\}$ for $x \in X_{2}^{+} \backslash\{\mathbf{0}\}$ and $\omega(x)=\{\mathbf{0}\}$ for $x \in X_{1}^{+}$. If $r_{2} a_{12} \leq r_{1} a_{22}$, then $\omega(x)=\left\{E_{2}\right\}$ for $x=\left(x_{1}, x_{2}\right)$ with $x_{2}>0$ and $\omega(x)=\{\mathbf{0}\}$ for $x \in X_{1}^{+}$.

We notice that (i) through (iv) of the theorem follow from (ii) of Lemma 3.3, Lemma 3.2 , and Proposition 3.4, respectively.

It is easy to see that $s(A)=0$ if and only if

$$
a_{11}<0, \quad a_{22}<0, \quad \text { and } a_{11} a_{22}=a_{12} a_{22} .
$$

By calculating, we obtain that the principal eigenvectors of $A$ and $A^{\mathrm{T}}$ are $v=\left(a_{12}\right.$ $\left.a_{11}\right)^{-1}\left(a_{12},-a_{11}\right)$ and $w=\left(a_{21}-a_{11}\right)^{-1}\left(a_{21},-a_{11}\right)$ respectively. 
TheOREM 5.2. Suppose that the system (5.1) satisfies (5.4). Then exactly one of the following holds.

(i) If $r<\mathbf{0}$, then $\omega(x)=\{\mathbf{0}\}$ for $x \geq \mathbf{0}$.

(ii) If $r>\mathbf{0}$, then $\psi_{t}(x)$ tends to infinity as $t$ increases for $x \gg \mathbf{0}$. Moreover, $\omega(x)=$ $\left\{E_{i}\right\}$ for $x \in X_{i}^{+} \backslash\{\mathbf{0}\}$ in the case $r_{i}>0$ and $\omega(x)=\{\mathbf{0}\}$ for $x \in X_{j}^{+}$in the case $r_{j}=0$.

(iii) If $r=\mathbf{0}$, then $\omega(x)=\left\{\alpha\left(a_{12},-a_{11}\right)\right\}$ for $x \gg \mathbf{0}$, where $\alpha=\left(a_{12}-a_{11}\right)^{-1}$ $\left(\frac{x_{1}\left(a_{12}-a_{11}\right)}{a_{12}}\right)^{\frac{a_{21}}{a_{21}-a_{11}}} \times\left(\frac{x_{2}\left(a_{12}-a_{11}\right)}{-a_{11}}\right)^{-\frac{a_{11}}{a_{21}-a_{11}}}$; and $\omega(x)=\{\mathbf{0}\}$ for $x \in X_{1}^{+} \cup X_{2}^{+}$.

(iv) Assume $r_{1}>0$ and $r_{2}<0$. If $r_{2} a_{11}>r_{1} a_{21}$, then $\omega(x)=\left\{E_{1}\right\}$ for $x \gg \mathbf{0}$; if $r_{2} a_{11}<r_{1} a_{21}$, then $\psi_{t}(x)$ tends to infinity as $t$ increases for $x \gg \mathbf{0}$; if $r_{2} a_{11}=r_{1} a_{21}$, then $\omega(x)=\left\{\left(-\frac{r_{1}}{a_{11}}+\alpha \frac{a_{12}}{a_{12}-a_{11}}, \frac{-\alpha a_{11}}{a_{12}-a_{11}}\right)\right\}$ for $x \gg \mathbf{0}$, where $\alpha$ is the positive root of the equation $\left(-\frac{r_{1}}{x_{1} a_{11}}+\frac{\alpha a_{12}}{x_{1}\left(a_{12}-a_{11}\right)}\right)^{\frac{a_{21}}{a_{21}-a_{11}}} \times\left(\frac{-\alpha a_{11}}{x_{2}\left(a_{12}-a_{11}\right)}\right)^{-\frac{a_{11}}{a_{21}-a_{11}}}=1$; and in all cases, $\omega(x)=\left\{E_{1}\right\}$ for $x \in X_{1}^{+} \backslash\{\mathbf{0}\}$ and $\omega(x)=\{\mathbf{0}\}$ for $x \in X_{2}^{+}$.

(v) Assume $r_{1}<0$ and $r_{2}>0$. If $r_{1} a_{22}>r_{2} a_{12}$, then $\omega(x)=\left\{E_{2}\right\}$ for $x \gg \mathbf{0}$; if $r_{1} a_{22}<r_{2} a_{12}$, then $\psi_{t}(x)$ tends to infinity as $t$ increases for $x \gg \mathbf{0}$; if $r_{1} a_{22}=r_{2} a_{12}$, then $\omega(x)=\left\{\left(\frac{\alpha a_{12}}{a_{12}-a_{11}},-\left(\frac{r_{2}}{a_{22}}+\frac{\alpha a_{11}}{a_{12}-a_{11}}\right)\right)\right\}$ for $x \gg \mathbf{0}$, where $\alpha$ is the positive root of the algebraic equation $\left(\frac{\alpha a_{12}}{x_{1}\left(a_{12}-a_{11}\right)}\right)^{\frac{1 a_{21}-a_{11}}{a_{21}}} \times\left(-\frac{1}{x_{2}}\left(\frac{r_{2}}{a_{22}}+\frac{\alpha a_{11}}{a_{12}-a_{11}}\right)\right)^{-\frac{a_{11}}{a_{21}-a_{11}}}=1$; and in all cases, $\omega(x)=\{\mathbf{0}\}$ for $x \in X_{1}^{+}$and $\omega(x)=\left\{E_{2}\right\}$ for $x \in X_{2}^{+} \backslash\{\mathbf{0}\}$.

It is not difficult to see that (i) through (v) of Theorem 5.2 follow from (i) of Lemma 3.2 , (i) of Lemma 3.3, (iii) of Theorem 4.1, and Proposition 4.2 respectively.

Next, we consider the three-dimensional system:

$$
\begin{aligned}
& \dot{x}_{1}=x_{1}\left(r_{1}+a_{11} x_{1}+a_{12} x_{2}+a_{13} x_{3}\right), \\
& \dot{x}_{2}=x_{2}\left(r_{2}+a_{21} x_{1}+a_{22} x_{2}+a_{23} x_{3}\right), \\
& \dot{x}_{3}=x_{3}\left(r_{3}+a_{31} x_{1}+a_{32} x_{2}+a_{33} x_{3}\right) .
\end{aligned}
$$

From (iii) of Theorem 2.1, we know that $s(A)<0$ if and only if

$$
\left.\begin{array}{c}
a_{i i}<0(i=1,2,3), \quad a_{11} a_{22}-a_{12} a_{21}>0, \\
D_{3}=a_{11} a_{22} a_{33}+a_{21} a_{32} a_{13}+a_{31} a_{12} a_{23}-a_{13} a_{22} a_{31}-a_{23} a_{32} a_{11}-a_{33} a_{12} a_{21}<0
\end{array}\right\}
$$

and $s(A)=0$ if and only if

$$
a_{i i}<0(i=1,2,3), \quad a_{11} a_{22}-a_{12} a_{21}>0, \quad \text { and } \quad D_{3}=0 .
$$

When $I \subset\{1,2,3\}$ with $\# I<3,(5.5)_{\text {I }}$ has been discussed in Theorems 5.1 and 5.2. So we only have to consider the positive initial conditions. Let $I_{1}=\{1,2\}, I_{2}=\{2,3\}$, and $I_{3}=\{1,3\}$. The possible equilibria of (5.5) for which there exist exactly two positive components are $E_{I_{1}}, E_{I_{2}}, E_{I_{3}}$ given by $E_{I_{1}}=\left(x_{1}^{*}, x_{2}^{*}, 0\right), E_{I_{2}}=\left(0, u_{2}^{*}, u_{3}^{*}\right)$, and $E_{I_{3}}=$ $\left(v_{1}^{*}, 0, v_{3}^{*}\right)$ where $x_{i}^{*}(i=1,2)$ is defined in (5.3),

$$
\begin{aligned}
u_{2}^{*} & =\frac{r_{3} a_{23}-r_{2} a_{33}}{a_{22} a_{33}-a_{23} a_{32}}, & u_{3}^{*} & =\frac{r_{2} a_{32}-r_{3} a_{22}}{a_{22} a_{33}-a_{23} a_{32}}, \\
v_{1}^{*} & =\frac{r_{3} a_{13}-r_{1} a_{33}}{a_{11} a_{33}-a_{13} a_{31}}, & v_{3}^{*} & =\frac{r_{1} a_{31}-r_{3} a_{11}}{a_{11} a_{33}-a_{13} a_{31}} .
\end{aligned}
$$


If $s(A)<0$, then the possible positive equilibrium of $(5.5)$ is

$$
p^{*}=-A^{-1} r \text {. }
$$

If $r \notin \mathbf{R}_{+}^{3} \cup\left(-\mathbf{R}_{+}^{3}\right)$, we agree that $r_{i} \geq 0$ for $i \leq k$ and $r_{j}<0$ for $k<j \leq 3$, where $k=1$ or 2 . In other cases, we can make a transposition of the variables $x_{1}, x_{2}$, and $x_{3}$ such that the new system belongs to one of the above systems.

Define

$$
\begin{aligned}
& \tilde{r}_{1}=\left(a_{22} a_{33}-a_{23} a_{32}\right)\left(r_{1}+a_{12} u_{2}^{*}+a_{13} u_{3}^{*}\right), \\
& \tilde{r}_{2}=\left(a_{11} a_{33}-a_{13} a_{31}\right)\left(r_{2}+a_{21} v_{1}^{*}+a_{23} v_{3}^{*}\right), \\
& \tilde{r}_{3}=\left(a_{11} a_{22}-a_{12} a_{21}\right)\left(r_{3}+a_{31} x_{1}^{*}+a_{32} x_{2}^{*}\right) .
\end{aligned}
$$

It is not difficult to prove that

$$
p^{*}=-(\operatorname{det} A)^{-1}\left(\tilde{r}_{1}, \tilde{r}_{2}, \tilde{r}_{3}\right) .
$$

THEOREM 5.3. Suppose that the system (5.5) satisfies (5.6). Then exactly one of the following holds.

(i) If $r \leq \mathbf{0}$, then $\omega(x)=\{\mathbf{0}\}$ for $x \geq \mathbf{0}$.

(ii) If $r>\mathbf{0}$, then $\omega(x)=\left\{p^{*}\right\}$ for $x \gg \mathbf{0}$.

(iii) Assume that $r_{1} \geq 0, r_{2} \geq 0$ with $r_{1}+r_{2}>0$ and $r_{3}<0$. If $\tilde{r}_{3}>0$, then $\omega(x)=\left\{p^{*}\right\}$ for $x \gg \mathbf{0}$; if $\tilde{r}_{3} \leq 0$, then $\omega(x)=\left\{E_{I_{1}}\right\}$ for $x \gg \mathbf{0}$.

(iv) Assume that $r_{1}>0, r_{2}<0$, and $r_{3}<0$. If $r_{1} a_{21}>r_{2} a_{11}$, then for $x \gg \mathbf{0}$, $\omega(x)=\left\{p^{*}\right\}$ in case $\tilde{r}_{3}>0$ and $\omega(x)=\left\{E_{I_{1}}\right\}$ in case $\tilde{r}_{3} \leq \mathbf{0}$; if $r_{1} a_{31}>r_{3} a_{11}$, then for $x \gg \mathbf{0}, \omega(x)=\left\{p^{*}\right\}$ in case $\tilde{r}_{2}>0$ and $\omega(x)=\left\{E_{I_{3}}\right\}$ in case $\tilde{r}_{2} \leq \mathbf{0}$; if $r_{1} a_{21} \leq r_{2} a_{11}$ and $r_{1} a_{31} \leq r_{3} a_{11}$, then $\omega(x)=\left\{\left(-\frac{r_{1}}{a_{11}}, 0,0\right)\right\}$ for $x \gg \mathbf{0}$.

Proof. (i) and (ii) of the theorem follow from Lemma 3.2 and (ii) of Lemma 3.3 respectively. We only prove (iv); (iii) can be proved in a similar way.

Suppose that $r_{1}>0, r_{2}<0$, and $r_{3}<0$. Then, if $r_{1} a_{21}>r_{2} a_{11}$, applying (iii) of Theorem 5.1, we obtain that (5.5) $)_{\mathrm{I}_{1}}$ contains the equilibrium $E_{I_{1}}$ in the interior of the face $H_{\mathrm{I}_{1}}^{+}$. From (5.8), we have $\tilde{r}_{3}=\left(a_{11} a_{22}-a_{12} a_{21}\right) r_{3}^{*}$, where $r_{3}^{*}$ is given in Proposition 3.4. Since (5.6) holds, $a_{11} a_{22}-a_{12} a_{21}>0$. Therefore, $\tilde{r}_{3}$ and $r_{3}^{*}$ keep the same sign. Applying Proposition 3.4, we conclude that for $x \gg \mathbf{0}, \omega(x)=\left\{p^{*}\right\}$ if $\tilde{r}_{3}>0$ and $\omega(x)=\left\{E_{I_{1}}\right\}$ if $\tilde{r}_{3} \leq 0$. Assume that $r_{1} a_{31}>r_{3} a_{11}$. Then we can prove the conclusion in the same way as above. Finally, if $r_{1} a_{21} \leq r_{2} a_{11}$ and $r_{1} a_{31} \leq r_{3} a_{11}$, then $r_{2}^{*}=a_{11}^{-1}\left(r_{2} a_{11}-a_{21} r_{1}\right) \leq 0$ and $r_{3}^{*}=a_{11}^{-1}\left(r_{3} a_{11}-a_{31} r_{1}\right) \leq 0$. Applying Proposition 3.4, we immediately conclude that $\omega(x)=\left\{\left(-\frac{r_{1}}{a_{11}}, 0,0\right)\right\}$ for $x \gg \mathbf{0}$.

We now deal with the case $s(A)=0$, that is, (5.7) holds. By computing, we can obtain the principal eigenvectors $v$ and $w$ of $A$ and $A^{\mathrm{T}}$ given by

$$
\begin{gathered}
v=\left(v_{1}, v_{2}, v_{3}\right)=\mu\left(a_{12} a_{23}-a_{13} a_{22}, a_{13} a_{21}-a_{23} a_{11}, a_{11} a_{22}-a_{12} a_{21}\right), \\
w=\left(w_{1}, w_{2}, w_{3}\right)=\nu\left(a_{21} a_{32}-a_{31} a_{22}, a_{31} a_{12}-a_{32} a_{11}, a_{11} a_{22}-a_{12} a_{21}\right),
\end{gathered}
$$

where $\mu=a_{12} a_{23}-a_{13} a_{22}+a_{13} a_{21}-a_{23} a_{11}+a_{11} a_{22}-a_{12} a_{21}$ and $\nu=a_{21} a_{32}-a_{31} a_{22}+$ $a_{31} a_{12}-a_{32} a_{11}+a_{11} a_{22}-a_{12} a_{21}$.

THEOREM 5.4. Suppose that the system (5.5) satisfies (5.7). Then exactly one of the following holds. 
(i) If $r<\mathbf{0}$, then $\omega(x)=\mathbf{0}$ for all $x \geq \mathbf{0}$.

(ii) If $r=\mathbf{0}$, then $\omega(x)=\{\alpha v\}$ with $\alpha=\left(\frac{x_{1}}{v_{1}}\right)^{w_{1}}\left(\frac{x_{2}}{v_{2}}\right)^{w_{2}}\left(\frac{x_{3}}{v_{3}}\right)^{w_{3}}$ for $x \gg \mathbf{0}$.

(iii) If $r>\mathbf{0}$, then $\psi_{t}(x)$ tends to infinity as $t$ increases for $x \gg \mathbf{0}$.

(iv) Assume that either $r_{1} \geq 0, r_{2} \geq 0$ with $r_{1}+r_{2}>0$ and $r_{3}<0$ or $r_{1}>0, r_{2}<0$ and $r_{3}<0$ with $r_{1} a_{21}>r_{2} a_{11}$. Then, for $x \gg \mathbf{0}, \psi_{t}(x)$ tends to infinity as $t$ increases if $\tilde{r}_{3}>0 ; \omega(x)=\left\{E_{I_{1}}\right\}$ if $\tilde{r}_{3}<0 ; \omega(x)=\left\{E_{I_{1}}+\alpha v\right\}$ if $\tilde{r}_{3}=0$, where $\alpha$ is the positive root of the following algebraic equation:

$$
\left(x_{1}^{*}+\alpha v_{1}\right)^{w_{1}}\left(x_{2}^{*}+\alpha v_{2}\right)^{w_{2}}\left(\alpha v_{3}\right)^{w_{3}}=x_{1}^{w_{1}} x_{2}^{w_{2}} x_{3}^{w_{3}} .
$$

(v) Assume that $r_{1}>0, r_{2}<0$, and $r_{3}<0$. Let $r_{1} a_{31}>r_{3} a_{11}$. Then for $x \gg \mathbf{0}, \psi_{t}(x)$ tends to infinity as $t$ increases if $\tilde{r}_{2}>0 ; \omega(x)=\left\{E_{I_{3}}\right\}$ if $\tilde{r}_{2}<0, \omega(x)=\left\{E_{I_{3}}+\alpha v\right\}$ if $\tilde{r}_{2}=0$, where $\alpha$ is the positive root of the algebraic equation

$$
\left(v_{1}^{*}+\alpha v_{1}\right)^{w_{1}}\left(\alpha v_{2}\right)^{w_{2}}\left(v_{3}^{*}+\alpha v_{3}\right)^{w_{3}}=x_{1}^{w_{1}} x_{2}^{w_{2}} x_{3}^{w_{3}} .
$$

Let $r_{1} a_{21} \leq r_{2} a_{11}$ and $r_{1} a_{31} \leq r_{3} a_{11}$. Then, for $x \gg \mathbf{0}, \omega(x)=\left\{\left(-\frac{r_{1}}{a_{11}}, 0,0\right)\right\}$ if either $r_{1} a_{21}<r_{2} a_{11}$ or $r_{1} a_{31}<r_{3} a_{11} ; \omega(x)=\left\{\left(-\frac{r_{1}}{a_{11}}, 0,0\right)+\alpha v\right\}$ if $r_{1} a_{21}=r_{2} a_{11}$ and $r_{1} a_{31}=$ $r_{3} a_{11}$, where $\alpha$ is the positive root of the following algebraic equation:

$$
\left(-\frac{r_{1}}{a_{11}}+\alpha v_{1}\right)^{w_{1}}\left(\alpha v_{2}\right)^{w_{2}}\left(\alpha v_{3}\right)^{w_{3}}=x_{1}^{w_{1}} x_{2}^{w_{2}} x_{3}^{w_{3}} .
$$

In order to show the applications of the results obtained in this section, we shall give two examples in the following.

EXAMPLE 1. Consider the three-dimensional Lotka-Volterra system

$$
\begin{aligned}
& \dot{x}_{1}=x_{1}\left(15-10 x_{1}+2 x_{2}+3 x_{3}\right), \\
& \dot{x}_{2}=x_{2}\left(-1+x_{1}-20 x_{2}+x_{3}\right), \\
& \dot{x}_{3}=x_{3}\left(r_{3}+4 x_{1}+x_{2}-5 x_{3}\right) .
\end{aligned}
$$

In the system (5.8), the community matrix and the auto-increase coefficients are

$$
A=\left(\begin{array}{ccc}
-10 & 2 & 3 \\
1 & -20 & 1 \\
4 & 1 & -5
\end{array}\right) \quad \text { and } r=\left(\begin{array}{c}
15 \\
-1 \\
r_{3}
\end{array}\right)
$$

It is easy to see that (SM) holds, $a_{11} a_{22}-a_{12} a_{21}=198>0$, and $\operatorname{det} A=-729<0$. Therefore, (5.6) is true. Using the expression (5.3), we compute that $\tilde{r}_{3}=198 r_{3}+1197$. Applying (iv) of Theorem 5.3 or Proposition 3.4, we conclude that for $x \gg \mathbf{0}, \psi_{t}(x)$ converges to $-A^{-1} r \gg \mathbf{0}$ if $r_{3}>-\frac{1197}{198}$ and $\psi_{t}(x)$ converges to $\left(\frac{1192}{198}, \frac{5}{198}, 0\right)$ if $r_{3} \leq-\frac{1197}{198}$.

EXAMPLE 2. Consider the three-dimensional Lotka-Volterra system:

$$
\begin{aligned}
& \dot{x}_{1}=x_{1}\left(2-x_{1}+\frac{1}{2} x_{2}+\frac{1}{2} x_{3}\right), \\
& \dot{x}_{2}=x_{2}\left(-1+\frac{1}{2} x_{1}-x_{2}+\frac{1}{2} x_{3}\right), \\
& \dot{x}_{3}=x_{3}\left(-1+\frac{1}{2} x_{1}+\frac{1}{2} x_{2}-x_{3}\right) .
\end{aligned}
$$

In this system, the community matrix and the auto-increase coefficients are

$$
A=\left(\begin{array}{ccc}
-1 & \frac{1}{2} & \frac{1}{2} \\
\frac{1}{2} & -1 & \frac{1}{2} \\
\frac{1}{2} & \frac{1}{2} & -1
\end{array}\right) \quad \text { and } \quad r=\left(\begin{array}{c}
2 \\
-1 \\
-1
\end{array}\right)
$$


It is easy to see that (SM) holds, $a_{11} a_{22}-a_{21} a_{12}=\frac{3}{4}>0$, and $\operatorname{det} A=0$, that is, (5.7) holds. The principal eigenvector of $A$ is $v=\frac{1}{3}(1,1,1)$. Since $A^{\mathrm{T}}=A, w=v$. Therefore, $H(x)=x_{1} x_{2} x_{3}$ is an invariant function of $(5.9) .(2,0,0)$ is an equilibrium of $(5.9)$ and $r_{2}^{*}=r_{3}^{*}=0$ where $r_{2}^{*}$ and $r_{3}^{*}$ are given in Proposition 4.2. Applying Proposition 4.2 or (v) of Theorem 5.4, we know that for each fixed $x \gg \mathbf{0}, \omega(x)=\left\{(2,0,0)+\frac{\mu}{3}(1,1,1)\right\}$ where $\mu$ is the positive root of the cubic equation

$$
\mu^{3}+6 \mu=27 x_{1} x_{2} x_{3} .
$$

Solving (5.11), we obtain that

$$
\mu=\sqrt[3]{\frac{c-16}{2}+\sqrt{\frac{c}{4}(c-32)}}+\sqrt[3]{\frac{c-16}{2}-\sqrt{\frac{c}{4}(c-32)}}-2
$$

where $c=27 x_{1} x_{2} x_{3}$. We agree that if $c<32$ then the numbers

$$
\sqrt[3]{\frac{c-16}{2}+\sqrt{\frac{c}{4}(c-32)}} \text { and } \sqrt[3]{\frac{c-16}{2}-\sqrt{\frac{c}{4}(c-32)}}
$$

are conjugate to each other.

REMARK 5. With the help of Theorem 5.3, it is possible to give the complete classification for the asymptotic behavior of four-dimensional Lotka-Volterra systems with every solution bounded if (SM) is satisfied. We omit the detail here.

REMARK 6. Suppose that the community matrix $A=\left(a_{i j}\right)$ and the auto-increase coefficients $r$ are given and that $A$ satisfies the assumption (SM). Then, by Proposition 2.5 , the necessary condition for $(1.2)$ to be bounded is that $s(A) \leq 0$. From (iv) of Theorem 2.1 , we know that $s(A) \leq 0$ if and only if

$$
(-1)^{k}\left|\begin{array}{llll}
a_{11} & a_{12} & \cdots & a_{1 k} \\
a_{21} & a_{22} & \cdots & a_{2 k} \\
& \cdots & \cdots & \\
a_{k 1} & a_{k 2} & \cdots & a_{k k}
\end{array}\right|>0, \quad k=1,2, \ldots, n-1,
$$

and

$$
(-1)^{n} \operatorname{det} A \geq 0 \text {. }
$$

In the following, we shall provide an algorithm for determining the asymptotic behavior of (1.2) under the assumption (SM) and $s(A) \leq 0$.

First, we compute the leading principal minors of the community matrix $A$ and check the inequalities (5.11). Once (5.11) is satisfied, we immediately conclude that $s(A) \leq$ 0 , which includes all bounded systems. Next, we pick all nonnegative auto-increase coefficients, that is, we find the index subset $I=\left\{i: r_{i} \geq 0\right\}$. If $\# I=n$ or $r \leq \mathbf{0}$ then Lemmas 3.2 and 3.3 give the corresponding results of the asymptotic behavior for (1.2). Assume that $\# I<n$ and $r_{I}>\mathbf{0}$. Then, from (SM) and (iii) of Theorem 2.1, we obtain that $s\left(A_{I}\right)<0$ where $I=\left\{i_{1}<i_{2}<\cdots<i_{k}\right\}$ and $A_{I}=A\left(\begin{array}{cccc}i_{1} & i_{2} & \cdots & i_{k} \\ i_{1} & i_{2} & \cdots & i_{k}\end{array}\right)$. Thus, Theorem 2.4 implies that the linear algebraic equation

$$
A_{I} x_{I}+r_{I}=\mathbf{0}
$$


has a unique positive solution $c^{*}$. Solving Eq. (5.12), we obtain an equilibrium $c$ of (1.2) given by $c_{i}=c_{i}^{*}$ for $i \in I$ and $c_{i}=0$ for $i \notin I$. Such an equilibrium $c$ lies in Int $H_{I}^{+}$. Making the translation transformation (3.2), we get the system (3.3) and the new parameters $r_{k}^{*}=r_{k}+\sum_{j \in I} a_{k j} c_{j}^{*}$ for $k \in C(I)$. If all these parameters $r_{k}^{*}$ do not keep the same sign, we repeat this second procedure. By finite steps, we must obtain the system (3.3) satisfying the conditions of Proposition 3.4 or Proposition 4.2. Finally, applying Proposition 3.4 or Proposition 4.2, we can exactly obtain the asymptotic behavior of solutions of such a given system.

Before finishing this section, we present an example to show the application of this algorithm.

EXAMPLE 3. Consider the four-dimensional Lotka-Volterra system:

$$
\begin{aligned}
& \dot{x}_{1}=x_{1}\left(r_{1}-10 x_{1}+2 x_{2}+3 x_{3}+x_{4}\right), \\
& \dot{x}_{2}=x_{2}\left(r_{2}+x_{1}-20 x_{2}+x_{3}+x_{4}\right), \\
& \dot{x}_{3}=x_{3}\left(r_{3}+4 x_{1}+x_{2}-5 x_{3}+x_{4}\right), \\
& \dot{x}_{4}=x_{4}\left(r_{4}+x_{1}+x_{2}+x_{3}-a x_{4}\right),
\end{aligned}
$$

where $a \geq \frac{59}{81}$. It is easy to check that (SM) is true for the system (5.13). The community matrix given in (5.13) is

$$
A=\left(\begin{array}{cccc}
-10 & 2 & 3 & 1 \\
1 & -20 & 1 & 1 \\
4 & 1 & -5 & 1 \\
1 & 1 & 1 & -a
\end{array}\right)
$$

First, we compute the leading principal minors of $A$. By calculation, we have

$$
a_{11}=-10<0, \quad\left|\begin{array}{cc}
-10 & 2 \\
1 & -20
\end{array}\right|=198>0, \quad\left|\begin{array}{ccc}
-10 & 2 & 3 \\
1 & -20 & 1 \\
4 & 1 & -5
\end{array}\right|=-729<0
$$

and $\operatorname{det} A=9(81 a-59) \geq 0$ since $a \geq \frac{59}{81}$. Therefore, (5.11) holds.

If we let $r_{1}=20, r_{2}=r_{3}=r_{4}=-1$, then $I=\left\{i, r_{i} \geq 0\right\}=\{1\}$ and the system (5.13) has an equilibrium $c=(2,0,0,0)$ that is determined by $(5.12)$ with $I=\{1\}$. The corresponding parameters given in (3.3) are $r_{2}^{*}=1, r_{3}^{*}=7$, and $r_{4}^{*}=1$. By Propositions 3.4 and 4.2 , we deduce that all solutions of (5.13) with positive initial points converge to the unique equilibrium $-A^{-1} r$ if $a>\frac{59}{81}$ and that all solutions of (5.13) with positive initial points tend to infinity as $t$ increases if $a=\frac{59}{81}$.

If we choose $r_{1}=20, r_{2}=r_{4}=-3$, and $r_{3}=-9$, then $I=\{1\}$ and $r_{2}^{*}=r_{3}^{*}=r_{4}^{*}=-1$. Thus, Propositions 3.4 and 4.2 imply that the equilibrium $c=(2,0,0,0)$ attracts all positive conditions in $\mathbf{R}_{+}^{n}$.

If we choose $r_{1}=20, r_{2}=r_{4}=-2$, and $r_{3}=-8$, then $I=\{1\}$ and $r_{2}^{*}=r_{3}^{*}=r_{4}^{*}=0$. Applying Propositions 3.4 and 4.2, we conclude that every solution of (5.13) with a positive initial condition converges to $(2,0,0,0)$ if $a>\frac{59}{81}$ and that every solution of (5.13) with a positive initial condition converges to an equilibrium in $E_{+}=\{(2,0,0,0)+\mu v$ : $\mu>0\}$ where $v$ is the principal eigenvector of $A$. 
6. The generalization. In Secs. 4 and 5 , we have proved that every solution of a bounded cooperative Lotka-Volterra system with the assumption (SM) is convergent to an equilibrium. The aim of this section is to generalize this convergence result.

ThEOREM 6.1. Assume that the system (1.1) satisfies the following conditions:

(i) $f$ is $C^{1}$ and $\frac{\partial f_{i}}{\partial x_{i}}(x)>0$ for all $i \neq j$ and $x \in \mathbf{R}_{+}^{n}$ and $\frac{\partial f_{i}}{\partial x_{i}}(x)<0$ for each $i$ and $x$;

(ii) $D f(y) \leq D f(x)$ whenever $\mathbf{0} \leq x \leq y$;

(iii) every solution of (1.1) is bounded;

(iv) $s(D f(\mathbf{0})) \leq 0$.

Then every solution of (1.1) converges to an equilibrium. Furthermore, if $f(\mathbf{0})>\mathbf{0}$ or $f(\mathbf{0}) \leq \mathbf{0}$, the condition (iv) can be removed.

As soon as the corresponding results of Lemmas 3.2 and 3.3 are verified, the proof of this theorem is similar to those presented in Secs. 4 and 5. So we omit the details here.

Acknowledgment. The author is indebted to the referee whose suggestions led to an improvement of this paper.

\section{REFERENCES}

[1] B. S. Goh, Stability in models of mutualism, American Naturalist 113, 261-275 (1979)

[2] H. L. Smith, On the asymptotic behavior of a class of deterministic models of cooperating species, SIAM J. Appl. Math. 46, 368-375 (1986)

[3] H.-I. Freedman and H. L. Smith, Tridiagonal competitive-cooperative Kolmogorov systems, Differential Equations and Dynamical Systems (submitted)

[4] G. Karakostas and I. Györi, Global stability in job systems, J. Math. Anal. Appl. 131, 85-96 (1988)

[5] J. R. Gantmacher, The Theory of Matrices, Vol. II, Chelsea, New York, 1964

[6] W. A. Coppel, Stability and Asymptotic Behavior of Differential Equations, Heath, Boston, 1965

[7] M. W. Hirsch, Systems of differential equations which are competitive or cooperative I. Limit sets, SIAM J. Math. Anal. 13, 167-179 (1982)

[8] M. W. Hirsch, Systems of differential equations that are competitive or cooperative II. Convergence almost everywhere, SIAM J. Math. Anal. 16, 423-439 (1985)

[9] M. W. Hirsch, Stability and convergence in strongly monotone dynamical systems, J. reine angew. Math. 383, 1-53 (1988)

[10] J. F. Selgrade, Asymptotic behavior of solutions to single loop positive feedback systems, J. Differential Equations 38, 80-103 (1980)

[11] H. L. Smith and P. Waltman, The Theory of the Chemostat, Cambridge Univ. Press, 1995

[12] H. L. Smith, Competing subcommunities of mutualists and a generalized Kamke theorem, SIAM J. Appl. Math. 46, 856-873 (1986) 